\title{
Joint Coding of Multiple H.264 Video Programs
}

\author{
Luís Teixeira ${ }^{1}$ and Luís Corte-Real ${ }^{2}$ \\ ${ }^{1}$ Universidade Catolica Portuguesa, R. Diogo Botelho 1327, \\ 4169005 Porto, Portugal \\ Imt aporto.ucp.pt \\ ${ }^{2}$ FE.UP / INESC Porto, R. Roberto Frias, \\ 4250005 Porto, Portugal \\ Irealainescporto.pt
}

\begin{abstract}
This paper presents a joint coding scheme for multiple H.264/AVC video programs. Channel bandwidth is divided among different programs according its complexity. Several complexity metrics are assessed. Initial results are presented and discussed.
\end{abstract}

Keywords: Joint Coding, video quality, H.264.

\section{Introduction}

Video services, such as digital television, video on demand, Internet video, are been offered by a broad range of video content suppliers. Each service can contain several programs. By a program we refer to one or more bit streams that are used to represent the video content and associated audio content. The diffusion of the video services over data communications and broadcasting networks requires considerable substantial bandwidth.

Examples of broadcasting system varies from packet-switched data communication networks to streaming broadcast networks including IP-based (Internet Protocol) networks such as the Internet, cellular networks, DVB-H (Digital Video BroadcastHandheld), DVB-T (Digital Video Broadcast-Terrestrial), DVB-S (Digital Video Broadcast-Satellite), DVB-S2 (Digital Video Broadcast-Satellite, specification March 2005), and DVB-C (Digital Video Broadcast-Cable) broadcasting networks.

The bandwidth required to broadcast an individual video program with highquality depends on the nature of the contents and it varies in time. As an example, football or basketball compressed programs typically need more bandwidth than news programs for the same equivalent subjective quality.

Traditional encoding systems allocate constant values for the bandwidth to each program; thus content providers have to careful chosen between bandwidth and program quality. This problem is one of the main constrains in systems such as wireless transmission and broadcasting systems. A joint coding system can dynamically allocate the available bandwidth among the different programs in an optimum way. This process reduces the mean data rate for each program.

The H.264/MPEG-4 Advanced Video Coding standard (H.264/AVC) [1] has accomplished a considerable progress regarding coding efficiency, substantially 
enhanced error robustness, and increased flexibility and scope of applicability relative to its predecessors [2]. It covers all frequent video applications ranging from mobile services and videoconferencing to IPTV, HDTV, and HD video storage [2].

In TV multi-program broadcast systems, the use of H.264/AVC standards allows economies in transmission bandwidth of programs, while providing a service with a higher quality regarding current systems [3].

In this paper, we propose a statistical multiplexing rate control scheme for H.264 coding of video sequences. To obtain uniform picture quality within multiple video programs, a novel complexity measure based on Structural Similarly Information is proposed, which can assess the coding complexity of the current frame regarding its perceptual quality. Furthermore, it can be implemented in each video encoder in a very simple way without resulting in an excessive increase in the computational power.

In Section 2, a brief introduction to H.264 is presented and in section 3 Video Quality Assessment is introduced. Finally, a scheme is proposed and results presented in the following sections.

\section{H.264 Video Coding Algorithm}

International study groups, VCEG (Video Coding Experts Group) of ITU-T (International Telecommunication Union - Telecommunication sector) and MPEG (Moving Picture Experts Group) [1] of ISO/IEC, have studied the video coding techniques for various applications of moving pictures since the beginning 1990s. With the goal of achieving better video compression ratios compared to previous standards, H.264 / MPEG-4 part 10 video coding standard was recently developed by the JVT (Joint Video Team) [1] consisting of experts from VCEG and MPEG. H.264 accomplishes relevant coding efficiency, simple syntax specifications, and seamless integration of video coding into all current protocols and multiplex architectures. As a result H.264 can support a variety of applications from video streaming to video broadcasting.

As in previous video standards, H.264 uses the same basic techniques i.e., transform for reduction of spatial correlation, quantization for bitrate control, motion compensated prediction for reduction of temporal correlation, entropy encoding for reduction of statistical correlation [1].

The encoded video data is organized into a layered structure. The sequence is the top of the hierarchy. It comprises one or more Group of Pictures (GOP). A GOP comprises a certain number of encoded images. The length of the GOP may be dictated by the random access requirements. It is used as a random access unit. A picture is a frame classified as intra, predicted or interpolated (bi-directional) according to the mode that was used to encode it. Images are divided into slices, slices are divided into sequence of macroblocks and each macroblock correspond to a region of pixels. The coder selects between intra and inters coding modes of each macroblock. In an Intracoded mode, a prediction block is created based on formerly reconstructed (but, unfiltered for deblocking) blocks. The residual signal between the current block and the prediction is then encoded. Inter coding (predictive or bi-predictive) is more efficient as it uses inter prediction of each block of sample values from some previously decoded pictures. 


\section{Video Quality Assessment}

Normally, to assess the video quality we can find, in the literature, metrics such as the Peak signal-to-noise ratio (PSNR) and the Mean Square Error (MSE), Sum of Squared Differences (SSD), Mean Absolute Difference (MAD), and Sum of Absolute Differences (SAD). These metrics can be determined by the following expressions:

$$
\begin{gathered}
M A D=\frac{1}{H W} S A D, \text { and } S A D=\sum_{i=0}^{H-1} \sum_{j=0}^{W-1}|p(i, j)-\hat{p}(i, j)| \\
M S E=\frac{1}{H W} S S D, \text { and } S S D=\sum_{i=0}^{H-1} \sum_{j=0}^{W-1}(p(i, j)-\hat{p}(i, j))^{2} \\
P S N R=10 \log _{10} \frac{A^{2}}{M S E}
\end{gathered}
$$

where $\mathrm{H}$ and $\mathrm{W}$ denote the image dimension (height and width of the image), $\mathrm{A}$ represents the maximum grey level of the image ( $\mathrm{A}=255$ for 8-bit representation), and $p(i, j)$ and $\hat{p}(i, j)$ represent the "original" and the processed image pixels at position (i, j). Among the major advantages of these characteristics are its simplicity (particularly in case of MAD and MSE) and its clear physical meaning. Nevertheless, these metrics present some problems in assessing signal fidelity [5][6] as they are based on pixel to pixel difference calculation and ignore human perception and the viewing condition. Ongoing work regarding the impact of coding distortion on the subjective quality is still under investigation [7], [8].

A new scheme for a class of quality metrics, known as structural similarity method (SSIM), has been proposed to models perception implicitly by taking into account the fact that the Human Visual System (HVS) is adapted for extracting structural information (relative spatial covariance) from images [5], [9]. This assumption is based on the fact that natural image signals are usually highly "structured" denoting that samples from natural image signals have strong dependency amongst themselves, and these dependencies contain significant information regarding the structures of the objects in the visual scene [5]. The SSIM index has been demonstrated in [4] to be an effective measurement of perceptual global degradations in natural images.

To calculate SSIM let us assume that $\mathbf{x}$ and $\mathbf{y}$ are the two images being compared, respectively. The local SSIM index measures the similarities of the local luminance $l(\mathbf{x}, \mathbf{y})$, contrast $c(\mathbf{x}, \mathbf{y})$, and structure $s(\mathbf{x}, \mathbf{y})$ using the following equation:

$$
S(\mathbf{x}, \mathbf{y})=l(\mathbf{x}, \mathbf{y}) \cdot c(\mathbf{x}, \mathbf{y}) \cdot s(\mathbf{x}, \mathbf{y})=\frac{2 \mu_{x} \mu_{y}+C_{1}}{\mu_{x}^{2}+\mu_{y}^{2}+C_{1}} \cdot \frac{2 \sigma_{x} \sigma_{y}+C_{2}}{\sigma_{x}^{2}+\sigma_{y}^{2}+C_{2}} \cdot \frac{\sigma_{x y}+C_{3}}{\sigma_{x} \sigma_{y}+C_{3}}
$$

where $\mu_{x}, \mu_{y}, \sigma_{x}, \sigma_{y}$ are the means and standard deviations of $\mathbf{x}$ and $\mathbf{y}$ respectively, $\sigma_{x y}$ is the cross correlation of $\mathbf{x}$ and $\mathbf{y}$ after removing their means, and $C_{1}, C_{2}$ and 
$C_{3}$ are small positive constants to avoid instability. The SSIM index is computed locally within a sliding window that moves pixel-by-pixel across the image, resulting in a SSIM map [9], [10]. The last step is to translate the quality map into a single quality index. This can be achieved by computing the mean value of local SSIM values or by other pooling methods [4]. Although its simplicity, the SSIM index performance is very good across a broad range of image and distortion types.
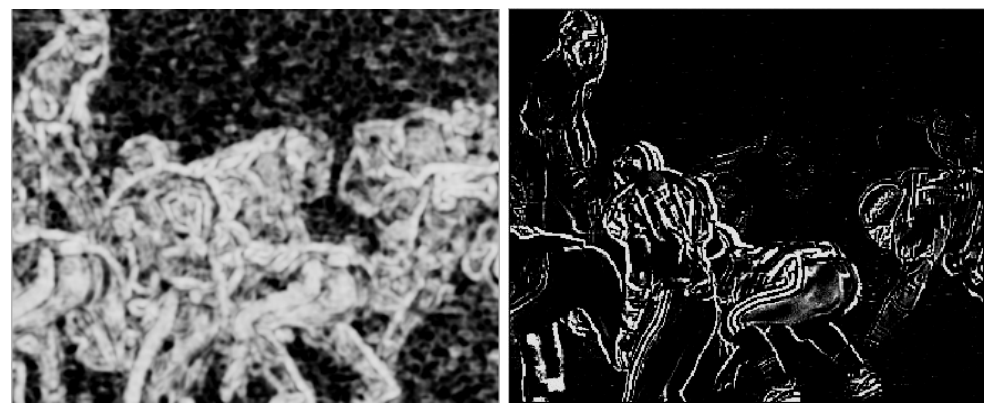

Fig. 1. Differences between structural and non-structural distortions for H.264, CBR 256kbps, frame 1, Football CIF sequence: (a) SSIM Distortion Map; (b) MAD Distortion Map

The performance of the SSIM index can be better comprehend if we analyzed the SSIM map between the original and the distorted images with the absolute error map as in the case of Fig. 1. The space-variant perceptual quality of the noisy image (e.g, the texture region of the grass field vs. the football players) has been well represented in the SSIM map, but not by the absolute error map. The artifacts in the grass from the football stadium field have been captured by the SSIM map, while the absolute error map fails to provide good predictions. SSIM index provides relevant local perceptual cues that can be of relevance for visual coding applications.

\section{Joint Coding Rate Control}

The proposed rate control and complexity measure application are discussed in this section. The Rate Control operate at different levels, specifically, sequence-level, frame level, and macroblock (MB)-level. Concerning joint coding of video sequences, sequence-level R-D control and optimization is performed to dynamically allocate the bandwidth among the video sequences to maximize the statistical multiplexing gain, that could be, for e.g., the use of the channel or the objective quality [11]:

In Figure 2, each video encoder generates encoded video and the resultant statistics. The joint rate module receives information regarding the relative complexities of each program and the channel buffer fullness. Each encoder changes its bit rate only when a new GOP begins.

Let $N_{g o p, p}$ designate the total number of frames in a group of picture (GOP) for the pth video program, $n_{i, j, p}\left(i=1,2, \cdots, j=1,2, \cdots, N_{g o p}, p=1,2, \cdots M\right)$ refer 


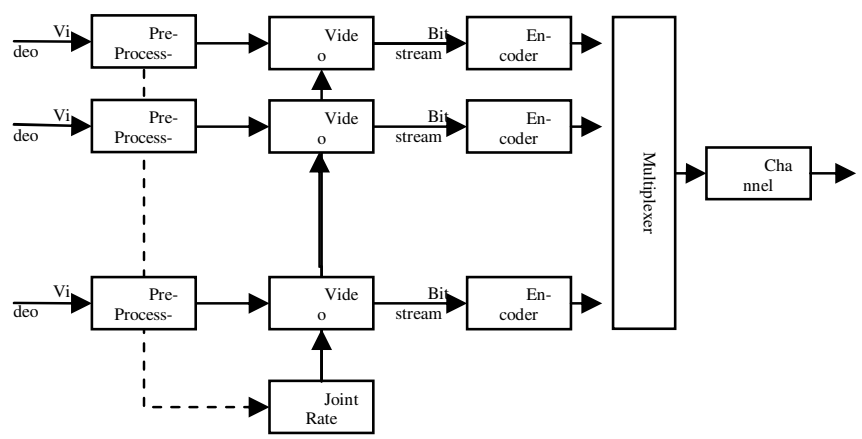

Fig. 2. Block Diagram for Joint Coding

to the $\mathrm{jth}$ frame in the ith GOP of the pth video program, $B_{c}\left(n_{i, j, p}\right)$ represent the occupancy of virtual buffer of the pth video program after encoding the jth frame in the ith GOP, BitRate $\left(n_{i, 0, p}\right)$ is the available bitrate at the ith GOP of the pth video program, and FrameRate is the predefined frame rate.

When the encoder starts to encode the ith GOP, the total bits allocated for the ith GOP of the pth video program can be determine by the following expression:

$$
T_{r}\left(n_{i, 0, p}\right)=\frac{\operatorname{BitRate}\left(n_{i, 0, p}\right)}{\text { FrameRate }} N_{\mathrm{GOP}}-\left.B_{c}\left(n_{i-1, N_{\mathrm{GOP}}, p}\right)\right|_{p=1,2, \ldots M} .
$$

If we consider the independent case this would be the available bandwidth. The aggregate bandwidth of all the video programs for the ith GOP can be determined as follows:

$$
T_{\text {aggregate }_{i}}=\sum_{p=1}^{M} T_{r}\left(n_{i, 0, p}\right) .
$$

The proposed joint rate control algorithm is based on the feedback model. Each encoder feeds the joint rate controller with coding statistics after finish encoding each picture. Simulation results in [11] indicate that the bit rate can be fits into a first order function of the SSIM metric. Thus, assuming a constant QP, the curve fitting results can be expressed as:

$$
R_{Q=\text { fixed }}=a+b \cdot \text { SSIM . }
$$

Where $\mathrm{R}$ denotes encoded bits, and $\mathrm{a}$ and $\mathrm{b}$ are zero-order and first-order constant coefficients extracted from curve fitting. Based on this linear relation, we proposed that by we can estimate the variation in quality for different bit rates in the neighborhood of a previous working point.

$$
\text { Quality }=\frac{R_{\text {new }}}{R_{Q=\text { fixed }}} \operatorname{SSIM}_{Q=\text { fixed }} .
$$


As our goal is to uniform the overall quality then we should make quality the same for each video sequence.

$$
\alpha=\frac{\operatorname{SSIM}_{Q=\text { fixed }}}{R_{Q=\text { fixed }}} .
$$

In our case we estimate $\alpha$ based on the previous encoded picture. For a 3 sources case, the following equations would be use

$$
\left\{\begin{array}{l}
T_{i, 1}=\frac{\alpha_{2} \cdot \alpha_{3}}{\alpha_{1} \cdot \alpha_{2}+\alpha_{1} \cdot \alpha_{3}+\alpha_{2} \cdot \alpha_{3}} \bullet T_{\text {aggregate }_{i}} \\
T_{i, 2}=\frac{\alpha_{1} \cdot \alpha_{3}}{\alpha_{1} \cdot \alpha_{2}+\alpha_{1} \cdot \alpha_{3}+\alpha_{2} \cdot \alpha_{3}} \bullet T_{\text {aggregate }_{i}} \\
T_{i, 3}=\frac{\alpha_{2} \cdot \alpha_{3}}{\alpha_{1} \cdot \alpha_{2}+\alpha_{1} \cdot \alpha_{3}+\alpha_{2} \cdot \alpha_{3}} \bullet T_{\text {aggregate }_{i}}
\end{array} .\right.
$$

\section{Simulations Results and Discussion}

We have implemented the proposed rate control scheme using the H.264 JM 10.2 encoder [12]. Simulations were performed using several video test sequences, with the same temporal duration, 10 seconds, and $352 \times 240$ resolution, representing different level of complexity, each one exhibiting different combined levels of spatial detail and amount of movement. Results presented here are with sequences Akiyo, Foreman and Football.

The test conditions under which our experiments were conducted are as follow: $\mathrm{MV}$ resolution $=1 / 4 \mathrm{pel} ; \mathrm{RDO}=\mathrm{ON} ;$ Search Range $=32 ;$ Frame Type $=$ IPPP and Reference Frame $=1$. The performance of our proposed scheme is evaluated in comparison with the original encoder JM 10.2. Two scenarios were studied. First each sequence encoded at fixed bit rate of $256 \mathrm{kbps}$ and then at fixed $512 \mathrm{kbps}$. Table 2 presents results for independent coding (Constant Bit Rate - CBR). GOP1 and GOP2 correspond to Intra Period of 4 and 10 frames respectively.

\begin{tabular}{|c|c|c|c|}
\hline Sequences & GOP & PSNRY (256kbps) & PSNRY (512kbps) \\
\hline \multirow[t]{2}{*}{ Akiyo } & IPPP_GOP1 & 37,90 & 41,82 \\
\hline & IPPP_GOP2 & 41,21 & 44,06 \\
\hline \multirow[t]{2}{*}{ Foreman } & IPPP_GOP1 & 31,13 & 34,40 \\
\hline & IPPP_GOP2 & 33,05 & 36,18 \\
\hline \multirow[t]{2}{*}{ Football } & IPPP_GOP1 & 28,52 & 31,90 \\
\hline & IPPP_GOP2 & 29,18 & 31,91 \\
\hline
\end{tabular}

Table 1. PSNR for Independent Coding 
With these three sequences 3 testing groups of two video sources were created. Several combinations were simulated with jointly combine 3 video streams: Akiyo is represented with letter A, Foreman with letter B and Football with letter C.

Table 2. Average Gaim for different strategic Joint Coding strategies (IPPP GOP1)

\begin{tabular}{llllll}
\hline BitRate & Metric & mux_psnr & mux_ssim & mux_sad & mux_ssd \\
\hline 256 & PSNR & $-0,27$ & $-0,28$ & $-0,69$ & $-0,57$ \\
256 & SSIM & $-0,85$ & $-0,85$ & $-2,49$ & $-1,90$ \\
512 & PSNR & $-0,01$ & $-0,03$ & $-0,29$ & $-0,14$ \\
512 & SSIM & 2,04 & 2,10 & 1,40 & 1,63 \\
\hline
\end{tabular}

Table 3. Average Gaim for different strategic Joint Coding strategies (IPPP GOP2)

\begin{tabular}{llllll}
\hline BitRate & Metric & mux_psnr & mux_ssim & mux_sad & mux_ssd \\
\hline 256 & PSNR & $-0,11$ & $-0,09$ & 0,01 & 0,05 \\
256 & SSIM & 1,28 & 1,39 & 1,40 & 1,38 \\
512 & PSNR & 0,20 & 0,21 & 0,26 & 0,27 \\
512 & SSIM & 3,55 & 3,52 & 3,30 & 3,00 \\
\hline
\end{tabular}

Table 2 and Table 3 compare results of using different ways to estimate coding complexity (PSNR, SSIM, SAD, and SSD). Results are presents as the variation in PSNR (dB) and SSIM. SSIM metric varies between 0 and 1 . We have scaled SSIM to make it more readable using the following equation:

\section{$100 \times \operatorname{SSIM}^{8}$}

SSIM approach present very interesting results regarding PSNR and the best results if we use SSIM quality metric to evaluate final quality. These are preliminary results and further studies need to be performed with more sources to confirm our first analysis. GOP pattern appears as an important factor

We have presented an algorithm for dynamic bandwidth allocation which allots the available bandwidth according to the needs of each video source and perceptual importance. Video sources with different combined levels of spatial detail and amount of movement benefit from this method. Simulation results show that bandwidth gains/quality improvements are more significant when heterogeneous sources are multiplexed together. An increased in subjective quality may be observed in football sequence while the decrease in Akiyo is rather small. Future work will include more sources.

\section{References}

[1] ITU-T and ISO/IEC JTC 1, Advanced Video Coding for Generic Audiovisual Services, ITU-T Rec. H.264 \& ISO/IEC 14496-10, Version 1 (May 2003); Version 2 (January 2004); Version 3 (with High family of profiles) (September 2004); Version 4 (July 2005)

[2] Wiegand, T., Sullivan, G.J.: The H.264/AVC Video Coding Standard \{Standards in a nutshell \}. IEEE Signal Processing Magazine 24(2) (March 2007) 
[3] Wiegand, T., Schwarz, H., Joch, A., Kossentini, F., Sullivan, G.J.: Rate-Constrained Coder Control and Comparison of Video Coding Standards. IEEE Transactions on Circuits and Systems for Video Technology 13(7), 688-703 (2003)

[4] Sheikh, H.R., Bovik, A.C.: Image Information and Visual Quality. IEEE Transactions on Image Processing 15(2), 430-444 (2006)

[5] Wang, Z., Bovik, A.C., Sheikh, H.R., Simoncelli, E.P.: Image quality assessment: From error visibility to structural similarity. IEEE Transactions on Image Processing 13(4), 600-612 (2004), http: / /www. cns . nyu . edu/ lcv/ssim/

[6] Sheikh, H.R., Sabir, M.F., Bovik, A.C.: A Statistical Evaluation of Recent Full Reference Image Quality Assessment Algorithms. IEEE Transactions on Image Processing 15(11), 3440-3451 (2006)

[7] Nemethova, O., Ries, M., Siffel, E., Rupp, M.: Subjective Evaluation of Video Quality for H.264 Encoded Sequences. In: Symposia TIC, Bratislava, Slovakia, October 24-26 (2004)

[8] Wolff, T., Ho, H.-H., Foley, J.M., Mitra, S.K.: H.264 coding artifacts and their relation to perceived annoyance. In: European Signal Processing Conference (2006)

[9] Brooks, A.C., Pappas, T.N.: Structural similarity quality metrics in a coding context: exploring the space of realistic distortions. In: IEEE International Conference on Acoustics, Speech, and Signal Processing, Honolulu, Hawaii, April 15-20, pp. 869-872 (2007)

[10] Wang, Z., Bovik, A.C., Simancelli, E.P.: Structural Approaches to Image Quality Assessment. In: Bovik, A. (ed.) The Handbook of Image and Video Processing, 2nd edn. Academic Press, London (2005)

[11] Perkins, M., Arnstein, D.: Statistical multiplexing of multiple MPEG-2 video programs in a single channel. SMPTE Journal 104(9), 596-599 (1995)

[12] Teixeira, L., Corte-Real, L.: Coding Multiple H.264 Video Streams using SSIM as Quality Metric. In: 2nd IEEE International Workshop on Multimedia Analysis and Processing (IMAP), St. Thomas, US Virgin Island, USA, August 4-7 (2008) (to be presented)

[13] Joint Video Team (JVT), H.264/Advanced Video Coding reference software version 10.2 (2006), http: / / iphome.hhi.de/suehring/tml/ 